\title{
THE POSSIBILITY OF INTERFERENCE SUPPRESSION BY CORRELATION RECEIVER APPLIED TO MARKER LOCALIZATION
}

\author{
Peter Vestenický ${ }^{1, \bowtie}$ and Martin Vestenický ${ }^{2}$ \\ ${ }^{1}$ University of Žilina, Faculty of Electrical Engineering and Information Technology \\ Department of Control and Information Systems, \\ Univerzitná 8215/1, 01026 Žilina, Slovakia \\ peter.vestenicky@fel.uniza.sk \\ ${ }^{2}$ University of Žilina, Faculty of Electrical Engineering and Information Technology \\ Department of Multimedia and Information-Communication Technologies \\ Univerzitná 8215/1, 01026 Žilina, Slovakia \\ martin.vestenicky@fel.uniza.sk
}

\begin{abstract}
The actual applications of the radiofrequency identification (RFID) systems in industry are focused not only on the identification but also on the localization of the RFID transponders. The special type of the RFID transponders is used to localize and to identify the underground facility networks such as pipes and cables. In such applications the RFID transponders are called markers. The paper describes an analysis of the correlation receiver for RSSI based localization of the inductive coupled RFID markers. The analysis is performed by the modeling of the localization device and the marker in Matlab - Simulink software. The aim of the analysis is to examine the ability of the correlation receiver to suppress the interfering signals from industrial sources, for example from the long wave telemetry transmitters which have their working frequencies in the same band as the working frequencies of the markers.
\end{abstract}

Keywords: Localization, RFID, marker, correlation receiver, interference suppression

\section{Introduction}

The RFID (Radiofrequency Identification) technology is very important part of the Industry 4.0 processes [1]. The passive RFID transponders working in LF (Low Frequency) or HF (High Frequency) bands (typically $125 \mathrm{kHz}$ or 13.56 $\mathrm{MHz}$ ) are inductively coupled with the reader. This fact causes that they work in near electromagnetic field where the intensity of the field depends inversely proportional on the 3rd power of distance. On the other side the transponders working in UHF (Ultra High Frequency) band (typically $868 \mathrm{MHz}$ ) work in the far electromagnetic field where the intensity of the field depends inversely proportional only on the 1st power of distance. But if the underground facilities such as water and gas pipes or power and telecommunication cables have to be identified and localized the only selection is to use the LF band because the properties of (especially wet) soil do not allow the use of HF nor UHF band. Note that the transponders are placed under the terrain surface near the underground facility. Therefore, the RFID transponders - markers - for marking such underground facilities work in LF band. The working frequencies of the markers are $77.0 \mathrm{kHz}$ for CATV cables, $83.0 \mathrm{kHz}$ for gas pipes, $101.4 \mathrm{kHz}$ for telecommunication cables, $121.6 \mathrm{kHz}$ for waste water pipes, $145.7 \mathrm{kHz}$ for water pipes, $134.0 \mathrm{kHz}$ (EU) or $169.8 \mathrm{kHz}$ (USA) for electrical power cables. The markers can be used only for localization purposes when they contain only a simple tuned LC circuit (single bit transponder) or for localization and identification when they must also contain an identification chip [2]. The marker localization is performed from the terrain surface by localization device (locator) and it uses the RSSI [3] method.

Because the markers work in the near electromagnetic field the signal level from the markers falls rapidly with distance between the localization device and the marker and therefore the localization receiver must be able to work with small signal-to-noise ratios. Moreover, the LF band is occupied by other radio services such as telemetry transmitters for distribution of precise time (DCF 77) or for energetic networks control. In Central Europe the telemetry transmitters are located for example in Lakihegy - Hungary $(135.6 \mathrm{kHz}, 100 \mathrm{~kW})$, Burg - Germany $(139.0 \mathrm{kHz}, 100$ $\mathrm{kW})$ and Mainflingen - Germany $(129.1 \mathrm{kHz}, 100 \mathrm{~kW}$ and $77.5 \mathrm{kHz}, 50 \mathrm{~kW})$ [4]. The correlation receiver or its simpler implementation - matched filter is primarily intended to suppress the white noise in communication channel [5]. Therefore, the ability of the correlation receiver to suppress the deterministic interferences coming from the telemetry transmitters is examined in this paper. Initial study of this problem is published in [6].

\section{Related Works}

The correlation receiver or matched filter is being used as optimum receiver in noisy communication channels. The theory of correlation receiver [5] assumes that the noise in the channel is white noise (AWGN channel - Additive White Gaussian Noise). Therefore, the correlation receiver is widely adopted not only in the RFID applications.

The problems with noise in UHF RFID is studied in [7] where the authors measure a distribution of signal-to-noise ratio by using software defined radio receiver. The reduction of noise by the matched filter is described in [8] and [9], 
but in these papers the main application is focused on the pulse and continuous wave radars. The authors perform comparison of the matched filter and the wavelet transformation properties in noise reduction. Similar problems are solved in [10] where the matched filter is applied to reduce the noise in the pulse radar.

In the paper [11] the interference canceling matched filter is analyzed for application in mobile communication where the interferences are considered as interference generated by multiple channel users mixed with Gaussian noise. The paper [12] describes the analysis of self-interferences in UHF RFID readers. The self-interferences are generated by CW (continuous wave) carrier transmitter in the RFID reader and the carrier leaks into the RFID reader receiver channel. To suppress the self-interferences a broadband leakage canceler is used with respect to the noise in the transmitted carrier.

\section{Model of Marker Localization Device and Interferences}

The simulation model created in Matlab - Simulink software is shown in Figure 1. The antenna of marker localization device is simulated as serial resonant circuit consisting of the primary winding of the transformer LS_ANT_L_MKR with inductance $L_{1}$, capacitor CS_ANT and the variable damping resistor $R$. The marker is simulated as parallel resonant circuit consisting of the secondary winding of the transformer LS_ANT_L_MKR with inductance $L_{2}$, capacitor $C_{-} M K R$ and lossy resistor $R P_{-} M K R$ which is calculated the quality factor of the marker resonant circuit to be 90 . Both resonant circuits have set the resonant frequency to the value $125 \mathrm{kHz}$. Note that the resistors $R_{1}$ and $R_{2}$ have very high values $\left(10^{12} \Omega\right)$ and they are added into the marker model to enable a galvanic connection of the secondary winding with ground. Without these resistors the Simulink solver diverges.

The coupling between the locator antenna and marker coils is represented by the transformer coupling factor $k$ which (in reality) depends on distance $x$ between the antenna and marker according to the equations (1) and (2), where $M$ is the mutual inductance of the locator antenna and the marker coils, $N_{r}$ and $N_{m}$ are the number of the coils turns, $r_{r}$ and $r_{m}$ are the radiuses of coils and $\Theta$ is angle between the coils plane. For simplicity, we assume that both coils have identical axis, i.e. $\Theta=0^{\circ}$.

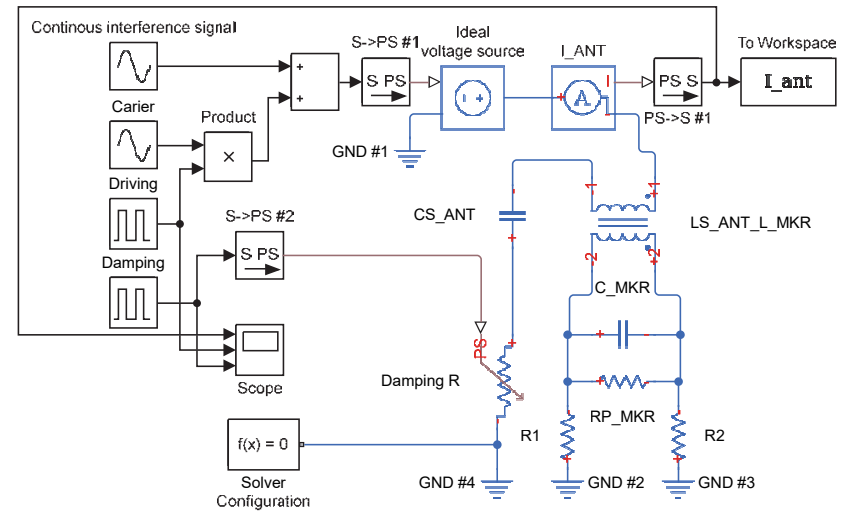

Figure 1: Model of the localization device, marker and interference source

$$
\begin{gathered}
k=\frac{M}{\sqrt{L_{1} \cdot L_{2}}} \\
M=\frac{\pi \cdot \mu_{0} \cdot N_{r} \cdot N_{m} \cdot r_{r}^{2} \cdot r_{m}^{2} \cos (\Theta)}{2 \cdot \sqrt{\left(r_{r}^{2}+x^{2}\right)^{3}}} \\
R=\left\{\begin{array}{cc}
10 & t<0.75 \mathrm{~ms}, t>0.79 \mathrm{~ms} \\
2 \cdot \sqrt{\frac{L_{1}}{C S_{-} A N T}} & t \in(0.75,0.79) \mathrm{ms}
\end{array}\right\}
\end{gathered}
$$

The marker parallel resonant circuit is excited by the harmonic carrier source with amplitude $A_{C}$ and frequency $f_{C}$ modulated by the driving signal (see Figure 1, Figure $2 \mathrm{c}$ and Table 1). After the end of the driving period the response of marker is received by the locator antenna as it is shown in the Figure $2 \mathrm{~b}$. The response is shaped as damped harmonic oscillations with frequency given by the marker resonant circuit. Between the driving period and the receiving period a small pause is placed which is necessary to damp the self-oscillations of the locator antenna serial resonant circuit by increasing of the value of the damping resistor $R$ which normally limits the current into the locator antenna $(R=10 \Omega)$ but during the damping pause its value is increased the quality factor of antenna resonant circuit to be $1 / 2$ (i. e. critical damping) what enables the fastest damping of the antenna self-oscillations, see equation (3) and Figure $2 \mathrm{~d}$. 

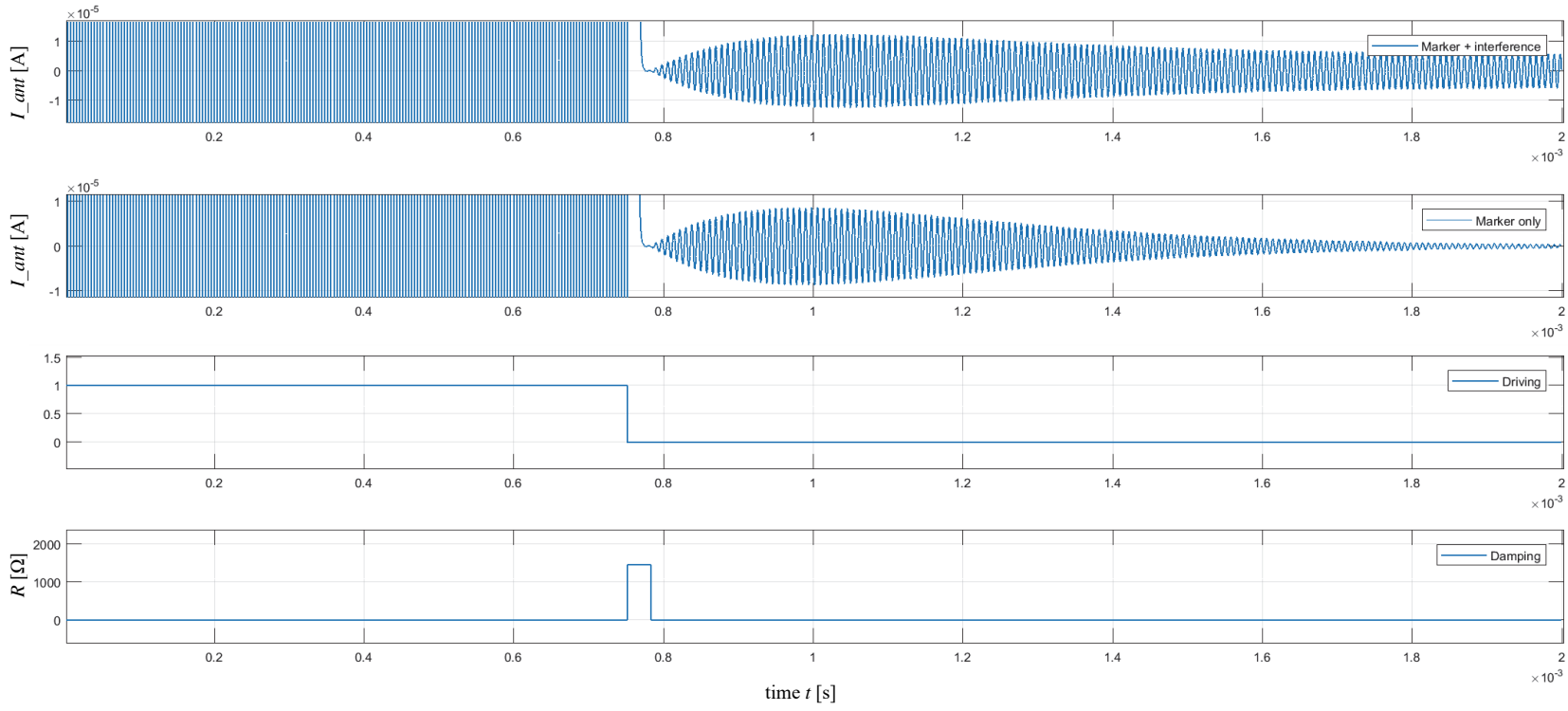

Figure 2a, b, c, d: Time courses of important signals in model

The continuous harmonic interference signal source in Figure 1 is added to the signal received from the marker response which is sensed as the current I_ANT flowing through the antenna circuit. The amplitude $A_{I N T}$ of this interference source is calculated by the equation (4) which is derived from the well-known Faraday's law where $\omega$ is angular frequency of the interference signal and $E$ is level of the electric field intensity generated by the telemetry transmitters. The equation (4) includes recalculation from the electric field intensity to the magnetic field intensity through a free space impedance which is approximately equal to $120 \pi \Omega$.

Table 1: Constant parameters of the model

\begin{tabular}{cccccc}
\hline Quantity & Value & Quantity & Value & Quantity & Value \\
\hline$N_{r}$ & 37 & $L_{1}$ & $925.16 \mu \mathrm{H}$ & $A_{C}$ & $12 \mathrm{~V}$ \\
$N_{m}$ & 37 & $L_{2}$ & $925.16 \mu \mathrm{H}$ & $f_{C}$ & $125 \mathrm{kHz}$ \\
$r_{r}$ & $0.1 \mathrm{~m}$ & $C S_{-} A N T$ & $1.7523 \mathrm{nF}$ & $R P \_M K R$ & $65.395 \mathrm{k} \Omega$ \\
$r_{m}$ & $0.1 \mathrm{~m}$ & $C_{-} M K R$ & $1.7523 \mathrm{nF}$ & $\Theta$ & $0^{\circ}$ \\
\hline
\end{tabular}

The level of intensity $E=85 \mathrm{~dB} \mu \mathrm{V} / \mathrm{m}$ was selected as a typical value in distance approximately $100 \mathrm{~km}$ from the long wave telemetry transmitter with effective isotropic radiated power $100 \mathrm{~kW}$ (see graph in [4]). The interference signal is added to the marker response signal as it is shown in Figure 2a. For next processing only the marker response signal in time $t>0.79 \mu$ s (Fig. 2a, b) is interesting and the signal is exported from the Simulink model into the Matlab workspace for the correlation analyses.

$$
A_{I N T}=\mu_{0} \cdot \omega \cdot N_{r} \cdot r_{r}^{2} \cdot \frac{10^{\frac{E}{20}-6}}{120}
$$

\section{Correlation Analysis of the Marker Responses with Addition of the Interference Signal}

The correlation receiver is based on calculation of correlation between the received marker response (which can be influenced by the interference signal) and the reference response of marker. The reference response was obtained from the model described in chapter 3 for distance $x=0.6 \mathrm{~m}$ and without interferences i.e. when $A_{I N T}=0 \mathrm{~V}$. The selection of distance $x$ for calculation of the reference response is not critical because the shape of maker response is practically independent on the distance and only its maximum amplitude is varying. For calculation of the correlation the wellknown Pearson formula (5) was used where $i a_{a} t_{i}$ is sample of the antenna current (i.e. received response of the marker), $i$ ant $r e f_{i}$ is reference sample of the antenna current and $n$ is total number of samples recorded during the receiving time period (from $0.79 \mathrm{~ms}$ up to $2 \mathrm{~ms}$ in Figure 2). 


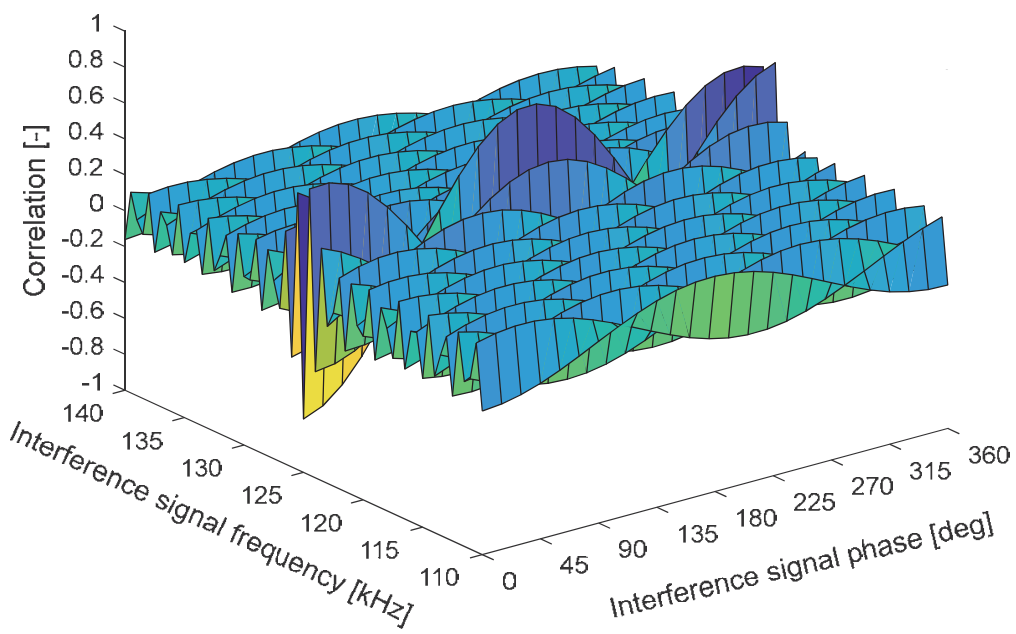

Figure 3: Correlations of the marker reference response and the harmonic interference signal without marker presence (distance $x=\infty$ )

Note that the application of the Pearson formula (5) is necessary to eliminate the dependency of the correlation on the distance between the marker and the locator antenna because the output of more frequently used implementation of the correlation receiver in form of the matched filter would be dependent on the maximum response amplitude and the maximum amplitude of the reference response, too.

The correlation analysis was performed for variable frequency of the interference signal in the range from $110 \mathrm{kHz}$ up to $140 \mathrm{kHz}$ with step $0.5 \mathrm{kHz}$ and for variable initial phase of the signal. The amplitude $A_{I N T}$ of harmonic interference signal in Simulink model in Figure 1 was calculated from the equation (4) and it is in the range from 47.6 $\mu \mathrm{V}$ up to $60.6 \mu \mathrm{V}$. The results are shown in 3D graph in Figure 3 where no marker is present i. e. the marker is in infinity distance. This analysis is important to evaluate the maximum of correlation without the marker presence but with presence of the interference signal. The maximum of correlation is approximately 0.7 .

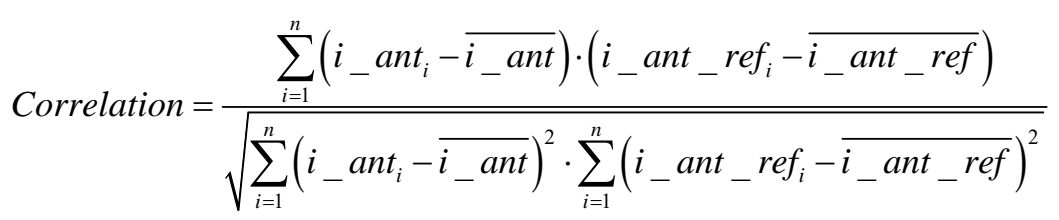

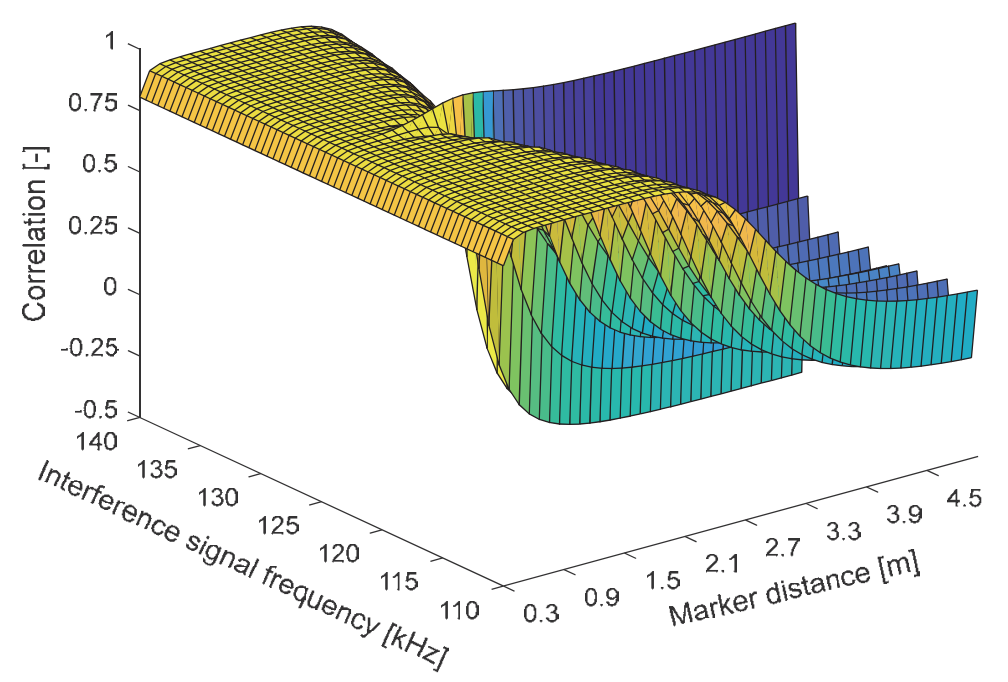

Figure 4: Correlation of the marker response and the reference response with addition of variable frequency harmonic interference signal and for variable distance between the marker and the locator antenna. 
Next analyses were performed with the presence of marker in distances $x$ from $0.3 \mathrm{~m}$ up to $5 \mathrm{~m}$ and the results are shown in 3D graph in Figure 4. Some cross - sections of this 3D graph are presented in Figure 5. These two graphs serve to evaluate the correlation receiver selectivity i. e. to evaluate if the receiver will be able to identify the marker presence even if the receiver is interfered by the unwanted deterministic signals.

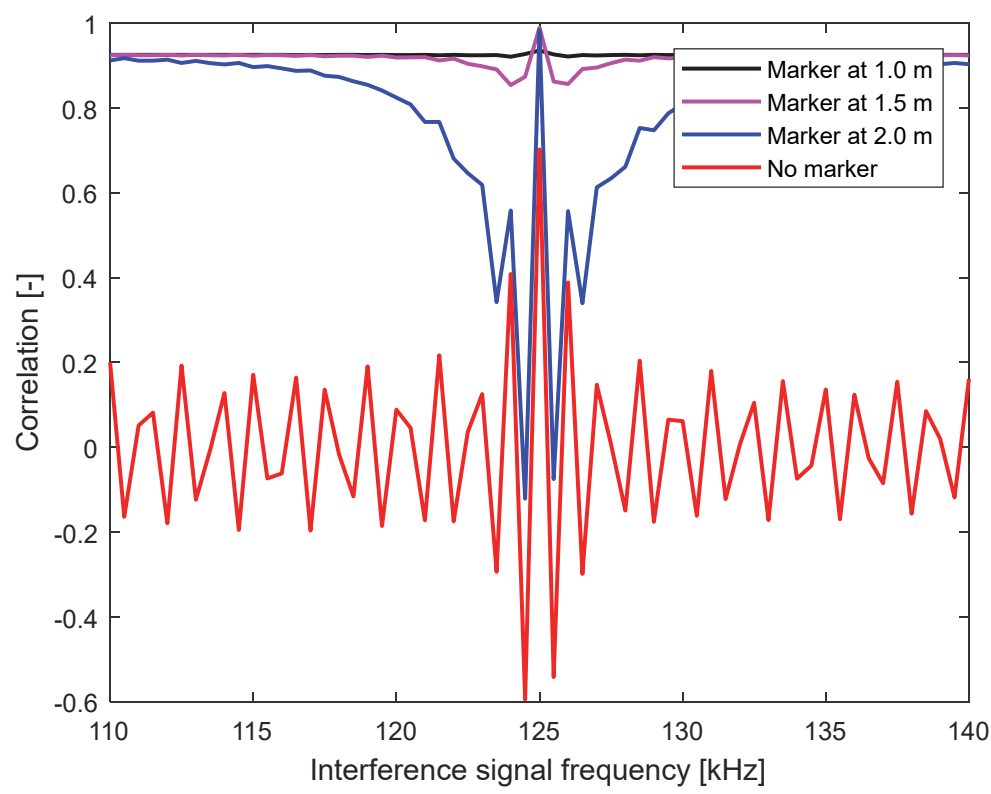

Figure 5: Correlations of the marker reference response and the marker response with addition of the harmonic interference signal. Distance between the marker and the locator antenna is parameter.

\section{Conclusion}

The presented analysis results demonstrate that the correlation receiver can be utilized to the marker localization by the RSSI method taking the selected limiting factors into account. The main limiting factor is the selectivity of receiver utilizing the marker response processing by the described method. The selectivity of the correlation receiver is sufficient to detect the marker presence in the distance approximately $1.5 \mathrm{~m}$ even if the harmonic interfering signal has the same frequency as the marker resonant frequency. If the frequency of interference signal differs from the marker resonant frequency the area of reliable marker detection is larger, up to $2 \mathrm{~m}$ from the locator antenna (Figure 4).

The future research will be focused on the investigation of the correlation receiver properties assuming the presence of combined stochastic and deterministic interferences (noise and telemetry transmitters), on the investigation of the quantizing noise of the receiver $\mathrm{A} / \mathrm{D}$ converter and integer arithmetic influence on the receiver selectivity and the accuracy of locator - marker distance estimation.

Acknowledgement: This work has been supported by the grant of Cultural and Educational Grant Agency of the Slovak Republic (KEGA) No. 038ŽU-4/2017: "Laboratory education methods of automatic identification and localization using radiofrequency identification technology."

\section{References}

[1] Peniak, P., Franeková, M.: Open communication protocols for integration of embedded systems within Industry 4. In: 20th International Conference on Applied Electronics, pp. 181-184. IEEE, Pilsen, Czech Republic, September 8th - 9th (2015). ISBN 978-8-0261-0385-1, ISSN 1803-7232.

[2] Finkenzeller, K.: RFID Handbook: Fundamentals and Applications in Contactless Smart Cards, Radio Frequency Identification and Near-Field Communication, 3rd Edition. John Wiley and Sons, Chichester (2010). ISBN 978-0470-69506-7.

[3] Brída, P., Machaj, J., Benikovský, J., Dúha, J.: An Experimental Evaluation of AGA Algorithm for RSS Positioning in GSM Networks. Electronics and Electrical Engineering 104(8), 113-118 (2010). ISSN 1392-1215.

[4] Wienold, H.: Radio Ripple Control. Europäische Funk-Rundsteuerung EFR GmbH, https://www.smartenergy.com/wp-content/uploads/Heinrich.pdf, last accessed 2018/08/07.

[5] Proakis, J., Salehi, M.: Digital Communication, 5th Edition. McGraw-Hill, New York, (2008). ISBN 978-0-07295716-7. 
[6] Mravec, T., Vestenický, P., Hruboš, M.: Application of correlation receiver on the RFID marker localization signals. In: 11th International Conference ELEKTRO 2016, pp. 440-444. IEEE, Štrbské Pleso, High Tatras, Slovak Republic, May 16th-18th (2016). ISBN 978-1-4673-8698-2.

[7] Blažević, Z., Solic, P., Skiljo, M., Stella, M.: Signal-to-noise ratio measurements and statistical characterization in Gen2 RFID. In: 2nd International Multidisciplinary Conference on Computer and Energy Science (SpliTech), pp. 1-4, Split, Croatia, 12th-14th July, (2017). ISBN 978-953-290-071-2.

[8] Islam, M.S., Chong, U.: Comparative Analysis of Matched Filter and Wavelets for Radar Noise Reduction. International Journal of Information and Electronics Engineering 4(2), 116-120 (2014). ISSN 2010-3719.

[9] Islam, M.S., Chong, U.: Noise reduction of continuous wave radar and pulse radar using matched filter and wavelets. EURASIP Journal on Image and Video Processing, vol. 2014, no. 1, pp. 1-9, December, (2014). ISSN 1687-5281.

[10] Sarkar, A., Pal, A.: Use of Matched Filter to Reduce the Noise in Radar Pulse Signal. International Journal of Recent Trends in Engineering and Research 2(2), 282-286, February (2016). ISSN 2455-1457.

[11] Slock, D.T.M., Trigui, H.: An Interference Cancelling Multichannel Matched Filter. In: Proceedings of GLOBECOM'96, IEEE Global Telecommunications Conference, London, United Kingdom, pp. 214-218, November 18th-28th (1996). ISBN 0-7803-3336-5.

[12]Lasser, G., Mecklenbräuker, C.F.: Self-interference noise limitations of RFID readers. In: IEEE International Conference on RFID, pp. 145-150, San Diego, CA, 15th-17th April, (2015). ISBN 978-1-4799-1937-6, ISSN 2374-0221. 